\title{
MicroRNA-125a-5p Affects Adipocytes Proliferation, Differentiation and Fatty Acid Composition of Porcine Intramuscular Fat
}

\author{
Jingjing Du ${ }^{1,+}$, Yan Xu ${ }^{1,+}{ }^{+}$, Peiwen Zhang ${ }^{1}$, Xue Zhao ${ }^{1}$, Mailin Gan ${ }^{1}$, Qiang Li ${ }^{2}$, Jideng Ma ${ }^{1}$, \\ Guoqing Tang ${ }^{1}$, Yanzhi Jiang ${ }^{3}$, Jinyong Wang ${ }^{4}$, Xuewei $\mathrm{Li}^{1}$, Shunhua Zhang ${ }^{1, *}$ and Li Zhu ${ }^{1, *}$ \\ 1 College of Animal Science and Technology, Sichuan Agricultural University, Chengdu 611130, China; \\ 18111521611@163.com (J.D.); yiyazhi@yahoo.com (Y.X.); sicau_zhangpeiwen@163.com (P.Z.); \\ 18227588896@163.com (X.Z.); 18299095425@139.com (M.G.); jideng_ma@sina.com (J.M.); \\ tyq003@163.com (G.T.); xuewei.li@sicau.edu.cn (X.L.) \\ 2 Sichuan Province General Station of Animal Husbandry, Chengdu 611130, China; 18398635864@163.com \\ 3 College of Life and Science, Sichuan Agricultural University, Chengdu 611130, China; jiangyz04@163.com \\ 4 Chongqing Academy of Animal Sciences, Chongqing 402460, China; kingyou@vip.sina.com \\ * Correspondence: zhangsh1919@163.com (S.Z.); zhuli7508@163.com (L.Z.); Tel.: +86-28-86291133 (S.Z. \& L.Z.) \\ + These authors contributed equally to this work.
}

Received: 18 December 2017; Accepted: 25 January 2018; Published: 7 February 2018

\begin{abstract}
Intramuscular fat (IMF) content and composition are considered crucial indicators of porcine meat quality. However, the molecular mechanism of porcine IMF development is still mostly unclear. Recently, new evidence suggested that microRNA (miRNAs) play important roles in porcine intramuscular adipogenesis. Previously, microRNA-125a-5p (miR-125a-5p) was identified as an important regulator of adipogenesis. In the present study, we found that the expression of miR-125a-5p is dynamically regulated during porcine intramuscular preadipocytes differentiation and that its expression levels in different porcine muscle tissues were negatively involved with IMF content. To investigate the potential function role of miR-125a-5p in IMF development, porcine intramuscular preadipocytes were collected and transfected with miR-125a-5p mimics, inhibitors, or a negative control (NC), respectively. The results showed that overexpression of miR-125a-5p promoted proliferation and inhibited differentiation of porcine intramuscular preadipocytes while inhibition of miR-125a-5p had the opposite effects. Furthermore, a luciferase reporter assay demonstrated that porcine kruppel like factor 3 (KLF13) is a target gene of miR-125a-5p during porcine intramuscular preadipocytes differentiation. Interestingly, porcine ELOVL fatty acid elongase 6 (ELOVL6), a regulator of fatty acid composition, was also identified as a target gene of miR-125a-5p during porcine intramuscular adipogenesis. Further studies show that miR-125a-5p overexpression reduced total saturated fatty acids (SFA) content and monounsaturated fatty acids (MUFA)/SFA ratios while having no significant impact on polyunsaturated fatty acids (PUFA)/SFA and n-6/n-3 ratios. Taken together, our results identified that miR-125a-5p may be a novel regulator of porcine intramuscular adipogenesis and the fatty acid composition of porcine IMF.
\end{abstract}

Keywords: intramuscular fat; fatty acid composition; differentiation; micoRNA-125a-5p; kruppel like factor 3

\section{Introduction}

Currently, pork production is a still growing industry on the international market [1]. Increasing evidence suggests that pork has become the most consumed meat worldwide accounting for more than $40 \%$ of the meat product in the world [1-3]. As living standards rise, however, more and more people pay close attention to porcine quality. Intramuscular fat (IMF) content and composition such as 
fatty acid are important meat quality characteristics, which not only play important roles in the quality of meat including muscle color, tenderness, water-holding capacity, juiciness and flavor of cooked meat, but are also closely associated with human health [4-7]. The quality of porcine meat is supposed to increase with higher IMF. An IMF content of $2-2.5 \%$ were even considered by some researchers as the optimal level in terms of sensory properties of porcine meat [6]. Decreasing dietary saturated fatty acids (SFA), increasing monounsaturated (MUFA) fatty acids, or reducing the polyunsaturated (PUFA) n-6/n-3 fatty acid ratio in meat may reduce the risk of cardiovascular disease and improve human health [8-10]. Nevertheless, the molecular mechanism regulating lipid accumulation and fatty acid composition in IMF among pork products remain poorly understood.

MicroRNAs (miRNAs) are an evolutionarily conserved group of endogenous, small non-coding regulatory molecules, which were demonstrated to take part in various biological processes such as cancer, mitochondrial biogenesis, cell proliferation, and differentiation by regulating target genes expression at post-transcriptional levels [11-13]. Recently, numerous studies reported that miRNA are involved in intramuscular preadipocyte development including proliferation and differentiation. For instance, Li et al. [14] found that miR-143 inhibited proliferation but promoted differentiation among bovine intramuscular preadipocytes. Guo et al. [15] suggested that up-regulated miR-145 expression inhibited porcine preadipocyte differentiation by negatively regulating insulin receptor substrate 1 (IRS1). Sun et al. [16] demonstrated that miR-34a enhanced adipogenesis in porcine intramuscular preadipocytes through the extracellular regulated MAP kinase (Erk) signaling pathway. Previously, miR-125a-5p was shown to mediate adipogenesis in 3T3-L1 cells and porcine preadipocytes $[17,18]$. However, whether miR-125a-p is associated with regulation of adipogenesis in IMF of pork meat is still unclear. Here, we show that expression levels of miR-125a-5p in porcine muscle tissues were negatively associated with IMF content. Function analysis demonstrated that miR-125a-5p may promote proliferation but may also inhibit differentiation of porcine intramuscular preadipocytes by regulating KLF13. In addition, further studies showed that miR-125a-5p could affect fatty acid composition in porcine intramuscular adipocytes by regulating ELOVL6, which suggests that miR-125a-5p may play an important role in porcine intramuscular adipogenesis.

\section{Results and Discussion}

\subsection{The Expression Levels of miR-125a-5p Was Negatively Associated with Porcine IMF Content}

miR-125a-5p has been identified as an important regulator of myogenesis and adipogenesis [18-20]. Recently, we found that miR-125a-5p was expressed at higher levels in psoas major muscle (PMM) than the longissimus dorsi muscle (LDM) (Figure 1A), of which PMM has a higher percentage of IMF than LDM [21,22] (Figure 1B). In order to investigate whether miR-125a-5p is involved in the formation of porcine intramuscular fat (IMF), we performed quantitative real-time PCR (qRT-PCR) to compare the expression levels of miR-125a-5p in different muscles from the Liangshan pig. The results show that the IMF content of tongue muscle (TON) and obliquus externus abdominis (OEA) is significantly greater than other muscles including gastrocnemius muscle (GAM), masseter (MAS), and peroneal longus (PEL) (Figure 1C). Previous studies indicated that diacylglycerol O-acyltransferase 2 (DGAT2) and ELOVL fatty acid elongase 6 (ELOVL6) are positively correlated with IMF in pork meat $[23,24]$. Consistent with that, the expression levels of DGAT2 and ELOVL6 showed a similar varying tendency with the content of IMF in the above muscle tissues (Figure 1D,E). Interestingly, qRT-PCR analysis showed that miR-125a-5p was expressed at higher levels in both PEL and MAS when compared to both TON and OEA (Figure 1F), suggesting that miR-125a-5p expression might be closely associated with IMF content. In order to further reveal the relationship between miR-125a-5p and IMF, we isolated porcine intramuscular preadipocytes to induce differentiation and then evaluated the temporal patterns of miR-125a-5p expression during porcine intramuscular adipogenesis. As shown in Figure 1G, the expression levels of miR-125a-5p gradually increased up to day 6 of intramuscular preadipocytes differentiation but then slightly decreased after that. This result showed a similar expression pattern with miR-425-5p, which have been demonstrated to 
inhibit differentiation and proliferation in porcine intramuscular preadipocytes [25]. Overall, these results suggest that miR-125a-5p may be negatively associated with porcine intramuscular adipogenesis.
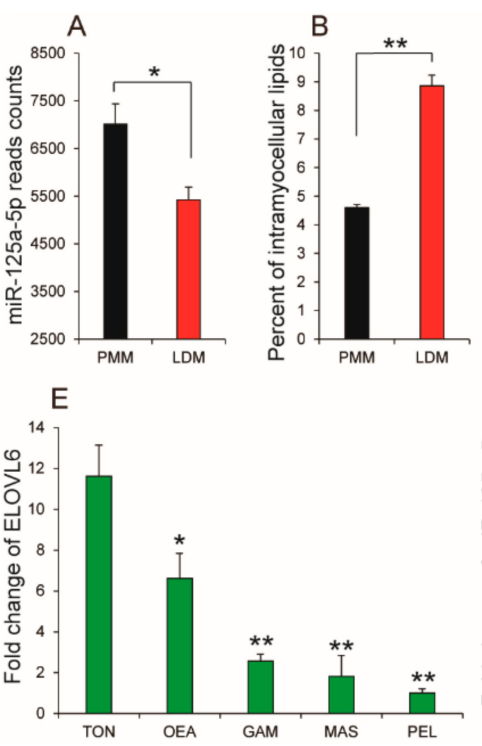
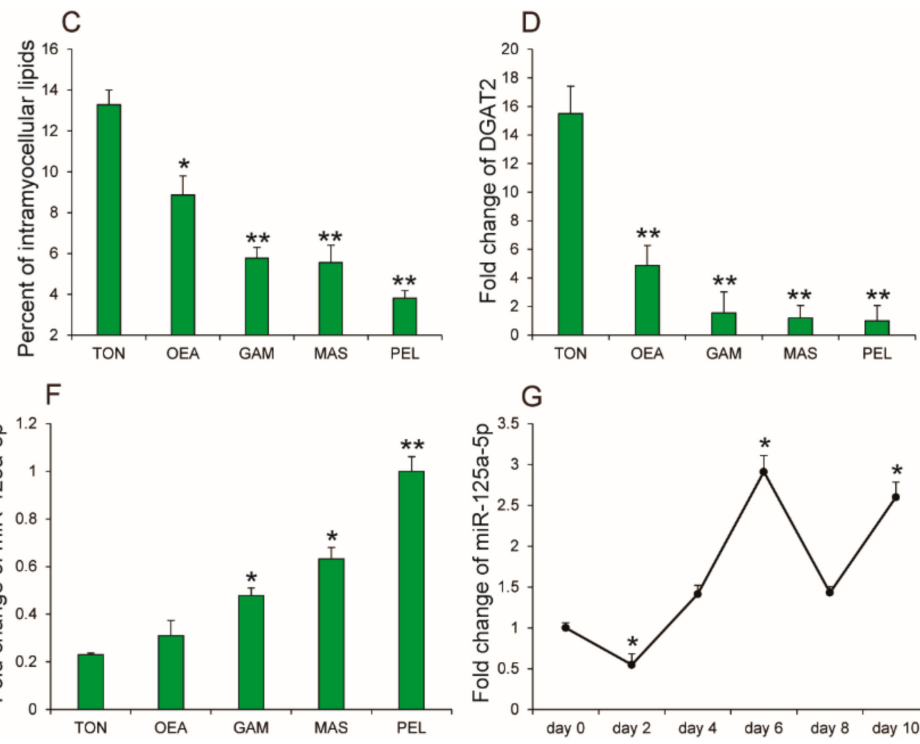

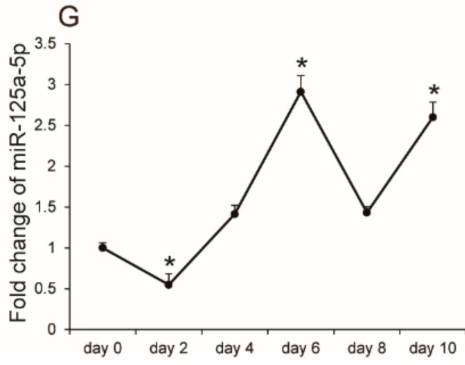

Figure 1. miR-125a-5p was negatively associated with porcine intramuscular fat (IMF) content. (A) The relative expression levels of miR-125a-5p in psoas major muscle (PMM) and longissimus dorsi muscle (LDM) ( $n=3$ per sample); (B) content of porcine IMF in PMM and LDM ( $n=3$ per sample); (C) IMF content in tongue (TON), obliquus externus abdominis (OEA), gastrocnemius muscle (GAM), masseter (MAS), and peroneal longus (PEL) ( $n=3$ per sample); (D-F) expression levels of diacylglycerol O-acyltransferase 2 (DGAT2), ELOVL fatty acid elongase 6 (ELOVL6) and miR-125a-5p in TON, OEA, GAM, MAS, and PEL ( $n=3$ per sample); (G) the relative expression levels of miR-125a-5p during porcine intramuscular preadipocytes differentiation ( $n=3$ per sample per time point). All results are presented as mean \pm SEM. ${ }^{*} p<0.05 ;{ }^{* *} p<0.01$.

\section{2. miR-125a-5p Promoted Proliferation of Porcine Intramuscular Preadipocytes}

Preadipocytes proliferation and differentiation are the basis on adipogenesis. To elucidate the potential function of miR-125a-5p in porcine intramuscular adipogenesis, we first explored the effect of miR-125a-5p on porcine intramuscular preadipocyte proliferation. As shown in Figure 2A, synthetic miR-125a-5p mimics inhibitors and the negative control (NC), which were transfected into porcine intramuscular preadipocytes, respectively. Additionally, miR-125a-5p mimics significantly increased the expression levels of miR-125a-5p by 13-fold in preadipocytes while endogenic expression of miR-125a-5p in preadipocytes was remarkably inhibited by transfecting with miR-125a-5p inhibitors. Subsequently, 5-ethynyl-2'-deoxyuridine (EdU) and Cell Counting kit 8 (CCK-8) analysis procedures were performed to evaluate the effect of miR-125a-5p on proliferation of porcine intramuscular preadipocytes. As shown in Figure 2B,C, EdU analysis suggested that, compared to the control group, miR-125a-5p overexpression significantly increased the ratio of EdU positive preadipocytes while inhibition of miR125a-5p strongly decreased the ratio of EdU positive preadipocytes. Meanwhile, CCK8 detection confirmed this effect, which showed that transfection miR-125a-5p mimics remarkably increased the total number of preadipocytes when compared with the control group. In contrast, the total number of preadipocytes transfected with miR-125a-5p inhibitors were significantly reduced compared with the control group (Figure 2D). These findings indicated that miR-125a-5p may promote porcine intramuscular preadipocyte proliferation. 

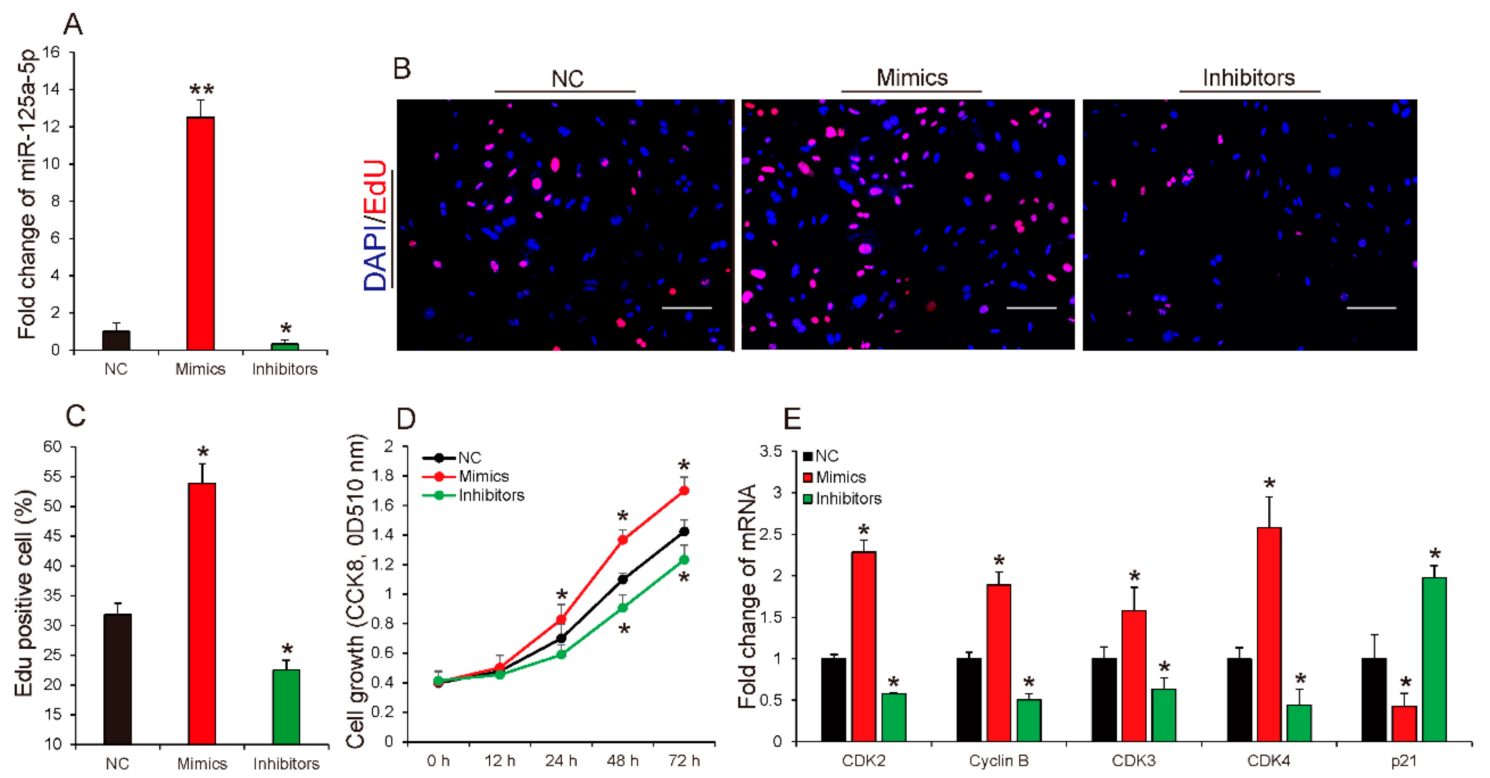

Figure 2. miR-125a-5p promoted proliferation of porcine intramuscular preadipocytes. (A) Relative expression levels of miR-125a-5p in porcine intramuscular preadipocytes transfected with miR-125a-5p mimics inhibitors or negative control (NC) $(n=3$ per treatment); (B,C) 5-ethynyl-2'-deoxyuridine (EdU) proliferation and (D) Cell Counting kit 8 (CCK-8) assays were performed to determine cell proliferation ( $n=6$ per treatment per time point); (E) the relative expression levels of cyclin-dependent kinases 2 (CDK2), Cell cycle protein B (Cyclin B), cyclin-dependent kinases 3 (CDK3), cyclin-dependent kinases 4 (CDK4), and cyclin-dependent kinase inhibitor $1 A$ (p21) when porcine intramuscular preadipocytes were transfected with mimics, inhibitors, or NC for 2 days ( $n=3$ per treatment). Scale bar, $100 \mu \mathrm{m}$. All results are presented as mean $\pm \mathrm{SEM} .{ }^{*} p<0.05 ;{ }^{* *} p<0.01$.

Cyclin-dependent kinases (CDKs) such as cyclin-dependent kinases 2 (CDK2) and cyclin-dependent kinases 3 ( $C D K 3$ ) have been recognized as key regulators of cell growth and proliferation in eukaryotes, which are required for G1-to-S phase transition in mammalian cells [26,27]. Cell cycle protein B $(C y c l i n B)$ is necessary for the progression of the cells into and out of the M phase of the cell cycle [28]. Conversely, overexpression of cyclin-dependent kinase inhibitor $1 A$ ( $p 21)$ may inhibit cell proliferation by causing cell cycle arrest, which is an inhibitor of CDKs [29]. To confirm whether miR-125a-5p mediation of the porcine intramuscular preadipocyte proliferation is associated with these factors, we compared gene expression in cells transfected, respectively, with miR-125a-5p mimics, inhibitors, or NC. As shown in Figure 2E, qRT-PCR analysis illustrated that overexpression of miR-125a-5p significantly up-regulated the expression levels of $C D K 2, C D K 3, C D K 4$ and Cyclin B, and inhibition of miR-125a-5p strongly down-regulated the expression of these genes. Additionally, overexpression or inhibition of miR-125a-5p caused a decrease or an increase in the expression of $p 21$ in porcine intramuscular preadipocytes, respectively. Taken together, all results suggest that miR-125a-5p might inhibit proliferation of porcine intramuscular preadipocytes.

\section{3. miR-125a-5p Inhibited Differentiation of Porcine Intramuscular Preadipocytes by Directly Targeting KLF13}

Next we identified the functional role of miR-125a-5p during porcine intramuscular preadipocytes differentiation. Transfection efficiency is shown in Figure 3A. We observed that overexpression of miR-125a-5p significantly inhibited intramuscular preadipocyte differentiation as determined by measuring the Oil Red O staining signal (Figure 3B) and triglycerides (TG) contents (Figure 3C) at day 8 of preadipocyte differentiation. By contrast, inhibition of miR-125a-5p had an opposite effect on the differentiation of porcine intramuscular preadipocytes compared with miR-125a-5p overexpression. In addition, by performing immunofluorescence of adiponectin and qRT-PCR analysis, we observed that transfection of miR-125a-5p mimics for 8 days significantly repressed the expression levels of adipogenic 
marker including adiponectin, CCAAT/enhancer binding protein $\alpha(C / E B P \alpha)$, peroxisome proliferator activated receptor $\gamma(P P A R \gamma)$, adipocyte fatty acid-binding protein 4 (FABP4), and fatty acid synthase (FASN) while transfection of miR-125a-5p inhibitors remarkably promoted the expression of these factors when compared with the control group (Figure 3D,E). These results suggest that miR-125a-5p may play a negatively functional role during porcine intramuscular preadipocyte differentiation. Lipid metabolism is a continuum from emulsification and uptake of lipids in the intestine to cellular uptake and transport to compartments such as mitochondria [30]. Increasing mitochondrial biogenesis in white adipose tissues can impair white adipocyte formation and enhance energy output in the form of heat [31,32]. Recently, Maude et al. [32] reported that miR-125b could affect mitochondrial biogenesis. These findings gave us a hypothesis that miR-125a-5p may accelerate the expenditure of porcine intramuscular adipocytes by increasing mitochondrial biogenesis. To test this hypothesis, porcine intramuscular preadipocytes were induced to differentiate for four days and were then transfected, respectively, with miR-125a-5p mimics, inhibitors, or NC. After four days of transfection, we measured mitochondrial content by calculating the ratio of mitochondrial DNA:nuclear DNA (mtDNA:nDNA). As shown in Figure 3F, overexpression of miR-125a-5p promoted mitochondrial biogenesis in adipocytes while inhibition of miR-125a-5p suppressed mitochondrial biogenesis in porcine intramuscular adipocytes when compared with the control group. Consistent with changes in mitochondrial biogenesis, genes related to mitochondrial biogenesis (Mterf1, Mitochondrial transcription termination factor 1; Tfam, Transcription factor A) (Figure 3G) and mitochondrial energy metabolism (Cox5b, Cytochrome c oxidase subunit Vb; TMEM70, Transmembrane protein 70; Cox8b, Cytochrome c oxidase subunit VIIIb; Uqcr10, Ubiquinol-cytochrome c reductase, complex III subunit X; and ATP6, ATP synthase F0 subunit 6) (Figure 3H) were expressed at higher or lower levels in adipocytes transfected with miR-125a-5p mimics or inhibitors, respectively. In addition, along with the increasing or decreasing of mitochondria, transfection of miR-125a-5p mimics significantly decreased the number of oil red $\mathrm{O}^{+}$cells (Figure 3I), triglyceride content (Figure 3J), and expression levels of two key transcriptional regulators of adipogenesis called C/EBP $\alpha$ and PPAR $\gamma$ (Figure 3K). The findings suggest that miR-125a-5p may accelerate IMF expenditure in pork products by enhancing mitochondrial biogenesis and energy metabolism. Taken together, these results suggest that miR-125a-5p could inhibited differentiation of porcine intramuscular preadipocytes.

Previously, several studies demonstrated that miRNAs take part in many biological processes by negatively regulating target genes through interaction with the $3^{\prime}$-untranslated regions ( $3^{\prime}$-UTR) of the target mRNAs [33-35]. To further explore detailed molecular mechanisms that miR-125a-5p inhibits porcine intramuscular preadipocytes differentiation, we predicted target genes of miR-125a-5p using TargetScan 7.1. Among the potential target genes, we observed that the 3'-UTR region of Kruppel-like factor 13 (KLF13) mRNA had a binding site for miR-125a-5p seed sequences and could also accelerate porcine adipocyte differentiation [36]. In addition, miR-125a-5p and KLF13 were reciprocally expressed during porcine intramuscular preadipocytes differentiation (Figure 3L). Overexpression or inhibition of miR-125a-5p downregulated or upregulated the expression of KLF13, respectively (Figure 3M). These findings lead us to hypothesize that KLF13 is a direct target of miR-125a-5p for negatively regulating differentiation of porcine intramuscular preadipocytes. To confirm that, luciferase reporter assays were performed. As shown in Figure 3N,O, we observed that transfection of miR-125a-5p mimics in porcine intramuscular preadipocytes directly repressed the luciferase activities of the wild-type KLF13 3'-UTR (WT-KLF13) reporter when compared to the control group while mutation of the miR-125a-5p binding site in porcine KLF13 3'-UTR completely stopped this response. This suggests that KLF13 is a direct target gene of miR-125a-5p during differentiation of porcine intramuscular preadipocytes. Additionally, Ji et al. [18] suggested that estrogen related receptor $\alpha(E R R \alpha)$ is a directly target gene of miR-125a-5p inhibiting porcine preadipocytes differentiation. We also found that miR-125a-5p overexpression significantly repressed $E R R \alpha$ while inhibition of miR-125a-5p enhanced its expression during differentiation of porcine intramuscular preadipocytes (Figure 3P). In summary, the data revealed that miR-125a-5p inhibited differentiation of porcine intramuscular preadipocytes by negatively regulating KLF13. 

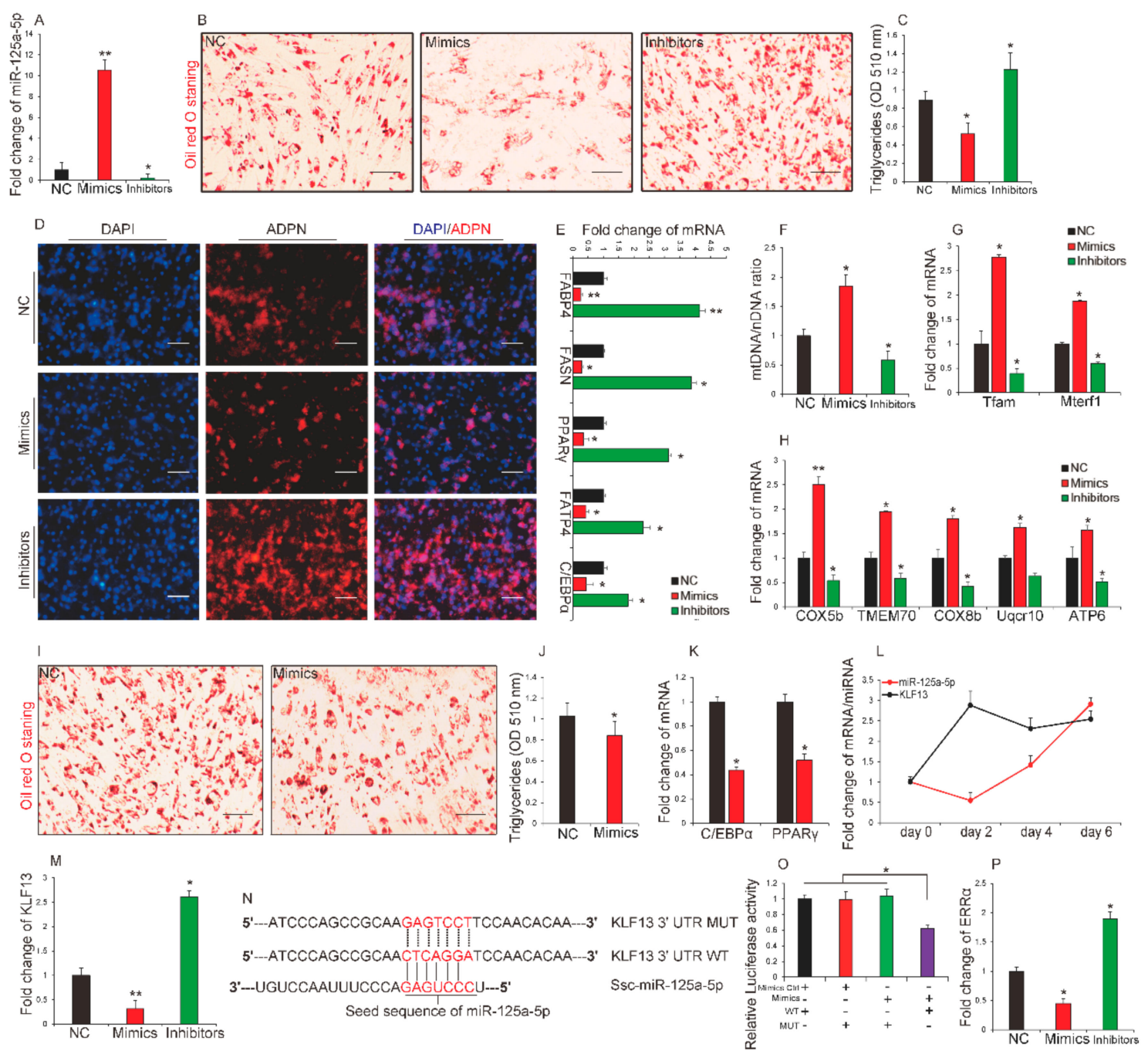

Figure 3. miR-125a-5p inhibited differentiation of porcine intramuscular preadipocytes by directly targeting Kruppel-like factor 13 (KLF13). After porcine intramuscular preadipocytes were induced differentiate and transfected with miR-125a-5p mimics, inhibitors or negative control (NC) for 8 days, (A) the relative expression levels of miR-125a-5p were measured by qRT-PCR ( $n=3$ per treatment); (B) Cells were stained with oil red O ( $n=3$ per treatment); (C) triglycerides content was measured by spectrophotometric analysis ( $n=4$ per treatment); (D) immunofluorescence of adiponectin was performed ( $n=3$ per treatment); (E) The relative expression levels of genes related to adipocytes differentiation ( $n=3$ per treatment). Additionally, porcine intramuscular preadipocytes that were induced differentiated for 4 days and were transfected respectively with miR-125a-5p mimics, inhibitors, or NC. (F) Mitochondrial content were evaluated by measuring the ratio of mtDNA:nDNA $(n=3$ per treatment); The relative expression levels of genes related to $(\mathbf{G})$ mitochondrial biogenesis and (H) mitochondrial energy metabolism ( $n=3$ per treatment); (I) cells were stained with oil red O $(n$ $=3$ per treatment); (J) triglycerides content was measured by spectrophotometric analysis $(n=3$ per treatment); (K) the relative expression levels of CCAAT/enhancer binding protein $\alpha(C / E B P \alpha)$, and peroxisome proliferator activated receptor $\gamma(P P A R \gamma)(n=3$ per treatment); $(\mathbf{L})$ The relative expression levels of miR-125a-5p and KLF13 during differentiation of porcine intramuscular preadipocytes $(n=3$ per treatment per time point); (M) The expression levels of KLF13 after cells were transfected with mimics, inhibitors, or NC ( $n=3$ per treatment); $(\mathbf{N})$ sequence alignment of Ssc-miR-125a-5p with 3'-UTR of porcine KLF13 mRNA; (O) Luciferase assays revealed the repressive effect of miR-125a-5p on the activity of KLF13 ( $n=7$ per treatment); (P) the expression levels of estrogen related receptor $\alpha(E R R \alpha)$ after cells were transfected with mimics, inhibitors or NC $(n=3$ per treatment). Scale bar, $100 \mu \mathrm{m}$. All results are presented as mean \pm SEM. ${ }^{*} p<0.05 ;{ }^{* *} p<0.01$. 


\section{4. miR-125a-5p Affected Fatty Acid Composition in Porcine Intramuscular Adipocytes}

Fatty acids are major contributors of meat nutritional value, which is associated with human health $[37,38]$. By performing luciferase reporter assays, we identified that porcine ELOVL6 is a target gene of miR-125a-5p, which has been shown to be a major regulator of fatty acid composition in pig tissue (Figure 4A,B). We then measured the difference in fatty acid composition after porcine intramuscular preadipocytes were induced to differentiate and transfected with miR-125a-5p mimics or negative control for eight days. As shown in Table 1, we found that miR-125a-5p overexpression had negative influence on C14:0, C15:0, C16:0, C16:1, C18:0, C18:1n9c, C20:2, C20:3n3, C20:4n6, C20:5n3, and significantly increased $\mathrm{C} 17: 1$ and $\mathrm{C} 20: 1$. However, no significant difference between the mimics group and the control group was observed for C6:0, C8:0, C10:0, C12:0, C17:0, C18:2n6c, C18:3n6, C20:0, C20:3n3, C20:3n6 and C22:6. Notably, these changes in content of C18:0 and C18:1 (including $\mathrm{C} 18: 1 \mathrm{n} 9 \mathrm{t}$ and $\mathrm{C} 18: 1 \mathrm{n} 9 \mathrm{c})$ between the mimics group and the negative group were consistent with prior results that miR-125a-5p overexpression significantly downregulated ELOVL6 expression in porcine intramuscular adipocytes (Figure 4C). Previous studies demonstrated that ELOVL6 could elongate $\mathrm{C} 12-\mathrm{C} 16$ to longer fatty acids and play an important role in the synthesis of stearic acid (C18:0) and oleic acid (C18:1) [39,40]. Meat is a primary source of the total amount of saturated fatty acids (SFA), which has been identified as a dietary risk factor closely associated with coronary heart disease and even various cancers [41,42]. Reducing the intake of SFA and increasing the intake of monounsaturated fatty acids (MUFA) can improve human health $[9,10]$. Further analysis found that miR-125a-5p overexpression significantly reduced total SFA content when compared with the control group (Figure 4D). However, overexpression had little impact on MUFA, SFA, and polyunsaturated fatty acids (PUFA) proportion in total fatty acids (Figure 4E). Furthermore, decreasing SFA content in mimics group when compared to the control group lowered levels of C14:0 and C18:0, which have a cholesterol rising effect [43] found in the mimics group but not the control group (Table 1). This suggests that miR-125a expression levels in porcine intramuscular adipocytes may be related to pork quality.

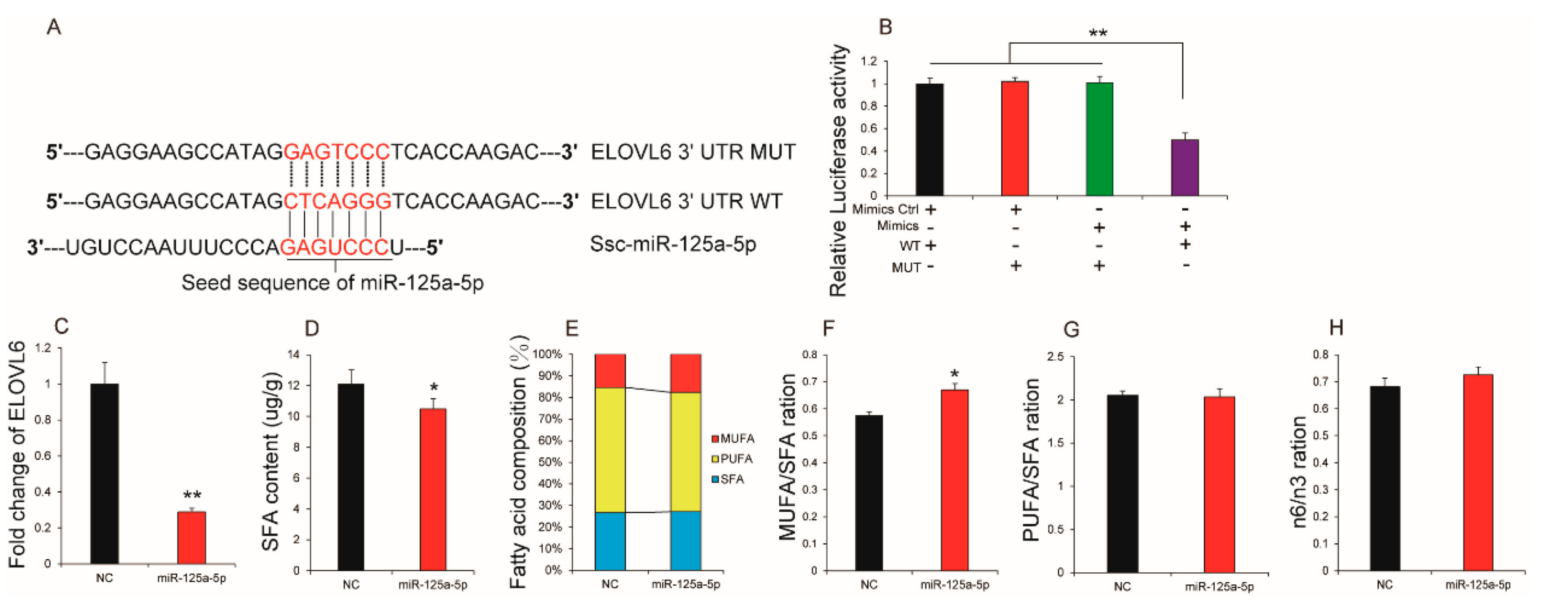

Figure 4. miR-125a-5p affected fatty acid composition in porcine intramuscular adipocytes. (A) Sequence alignment of Ssc-miR-125a-5p with 3'-UTR of porcine ELOVL6 mRNA; (B) Luciferase assays revealed the repressive effect of miR-125a-5p on the activity of ELOVL6 ( $n=7$ per treatment). After porcine intramuscular adipocytes were transfected with mimics or NC; (C) the expression levels of ELOVL6 $(n=3$ per treatment); (D) Saturated fatty acids (SFA) content in mimics group and control group $(n=3$ per treatment); (E) Fatty acid composition difference ( $n=3$ per treatment); (F) monounsaturated fatty acids (MUFA)/SFA ration ( $n=3$ per treatment); (G) polyunsaturated fatty acids (PUFA)/SFA ration $(n=3$ per treatment); $(\mathbf{H}) \mathrm{n}-6 / \mathrm{n}-3$ ration $(n=3$ per treatment). All results are presented as mean \pm SEM. $n=3$. ${ }^{*} p<0.05 ;{ }^{* *} p<0.01$. 
Table 1. miR-125a-5p regulate fatty acid composition in porcine intramuscular adipocytes.

\begin{tabular}{|c|c|c|c|}
\hline Fatty Acid & NC & Mimics & Up/Down \\
\hline C6:0 & 0.3489 & 0.3492 & down NS \\
\hline C8:0 & 0.5118 & 0.5119 & down NS \\
\hline C10:0 & 0.4812 & 0.4818 & down NS \\
\hline C12:0 & 0.7860 & 0.7838 & down ${ }^{N S}$ \\
\hline C13:0 & 0.0000 & 0.0000 & - \\
\hline C14:0 & 1.0003 & 0.8013 & down * \\
\hline C15:0 & 0.4443 & 0.3914 & down * \\
\hline C15:1 & 0.0000 & 0.0000 & - \\
\hline C16:0 & 3.0437 & 2.3119 & down * \\
\hline C16:1 & 2.2056 & 2.0297 & down * \\
\hline C17:0 & 0.4456 & 0.4022 & down NS \\
\hline C17:1 & 0.5076 & 0.7660 & up * \\
\hline C18:0 & 4.1666 & 3.5791 & down * \\
\hline $\mathrm{C} 18: 1 \mathrm{n} 9 \mathrm{t}$ & 0.0000 & 0.0000 & - \\
\hline $\mathrm{C} 18: 1 \mathrm{n} 9 \mathrm{c}$ & 3.9273 & 3.6161 & down * \\
\hline $\mathrm{C} 18: 2 \mathrm{n} 6 \mathrm{t}$ & 0.0000 & 0.0000 & - \\
\hline $\mathrm{C} 18: 2 \mathrm{n} 6 \mathrm{c}$ & 1.2904 & 1.0772 & down NS \\
\hline C18:3n3 & 0.0000 & 0.0000 & - \\
\hline C18:3n6 & 1.1420 & 1.0054 & down NS \\
\hline C20:0 & 0.8700 & 0.8703 & up ${ }^{\text {NS }}$ \\
\hline C20:2 & 1.3139 & 0.9447 & down * \\
\hline C20:1 & 0.3175 & 0.6081 & up * \\
\hline C20:3n3 & 0.7617 & 0.6041 & down* \\
\hline C20:3n6 & 1.1683 & 1.1799 & $u_{p}^{N S}$ \\
\hline C20:4n6 & 4.5844 & 3.9889 & down * \\
\hline C20:5n3 & 11.5488 & 8.9343 & down ** \\
\hline C21:0 & 0.0000 & 0.0000 & - \\
\hline C22:0 & 0.0000 & 0.0000 & - \\
\hline $\mathrm{C} 22: 1 n 9$ & 0.0000 & 0.0000 & - \\
\hline $\mathrm{C} 22: 2 \mathrm{n} 6$ & 0.0000 & 0.0000 & - \\
\hline C22:6 & 3.3151 & 3.5985 & up NS \\
\hline C24:0 & 0.0000 & 0.0000 & - \\
\hline C24:1 & 0.0000 & 0.0000 & - \\
\hline SFA & 12.0983 & 10.4831 & down * \\
\hline PUFA & 26.3246 & 21.3331 & down * \\
\hline MUFA & 6.9580 & 6.8200 & down NS \\
\hline n-6 & 8.3851 & 7.1514 & down * \\
\hline$n-3$ & 12.3105 & 9.8584 & down * \\
\hline
\end{tabular}

Saturated fatty acid (SFA) = C8:0 + C10:0 + C12:0 + C14:0 + C15:0 + C16:0 + C17:0 + C18:0 + C20:0 + C22:0; Monounsaturated fatty acid (MUFA) $=\mathrm{C} 14: 1+\mathrm{C} 16: 1+\mathrm{C} 18: 1+\mathrm{C} 20: 1+\mathrm{C} 24: 1 ;$ Polyunsaturated fatty acid $(\mathrm{PUFA})=$ $\mathrm{C} 18: 2+\mathrm{C} 18: 3+\mathrm{C} 20: 2+\mathrm{C} 20: 3+\mathrm{C} 20: 4+\mathrm{C} 20: 5+\mathrm{C} 22: 2+\mathrm{C} 22: 6 ; \mathrm{n}-3=\mathrm{C} 18: 3 \mathrm{n} 3+\mathrm{C} 20: 3 \mathrm{n} 3+\mathrm{C} 20: 5 \mathrm{n} 3 ; \mathrm{n}-6=\mathrm{C} 18: 2 \mathrm{n} 6 \mathrm{t}+$ $\mathrm{C} 18: 2 \mathrm{n} 6 \mathrm{c}+\mathrm{C} 18: 3 \mathrm{n} 6+\mathrm{C} 20: 3 \mathrm{n} 6+\mathrm{C} 20: 4 \mathrm{n} 6+\mathrm{C} 22: 2 \mathrm{n} 6 .{ }^{*} p<0.05 ;{ }^{* *} p<0.01 ; \mathrm{NS}$, no significant difference.

Additionally, compared to the content of particular fatty acids, nutritionists pay more attention to the PUFA/SFA ratio, the ratio of n-6/n-3, and meat products with higher ratios of PUFA and MUFA relative to SFA. A favorable balance between $n-6$ and n-3 in PUFA has a beneficial effect on disease prevention [44,45]. In order to further shed light on the relationship between miR-125a-5p and pork quality, we compared the ratios of PUFA/SFA, MUFA/ SFA, and n-6/n-3. The MUFA/SFA ratios were greater in the mimics group than the control group. However, no significant difference in PUFA/SFA and $n-6 / n-3$ ratios was observed between the mimics group and the control group (Figure $4 \mathrm{~F}-\mathrm{H}$ ). Taken together, these results suggest that miR-125a-5p could affect fatty acid composition in porcine intramuscular adipocytes. 


\section{Materials and Methods}

\subsection{Muscle Samples Collection}

Seven female LiangShan pigs that were reared in the same environment and fed the same diet were raised from birth to 180 days old. Subsequently, three pigs at a bodyweight 50-65 kg were selected for slaughter using the same strategy. In order to measure intramuscular fat (IMF) content and gene expression, muscle tissues including tongue muscle (TON), obliquus externus abdominis (OEA), gastrocnemius muscle (GAM), masseter (MAS), and peroneal longus (PEL) were quickly collected and stored at $-20{ }^{\circ} \mathrm{C}$ or $-80^{\circ} \mathrm{C}$, respectively. All experimental procedures were performed according to the guide for Animal Care and Ethics Committee of Sichuan Agricultural University, Sichuan (permit number DKY-S20143135, June 2014.).

\subsection{Porcine Intramuscular Preadipocytess Isolation and Culture}

According to previous reports [25,46,47], one female LiangShan Pig was sacrificed for porcine intramuscular preadipocytess isolation at three days. Briefly, Longissimus dorsi muscles (LDM) were aseptically isolated and washed three times using phosphate buffered saline (PBS, Gibco, Grand Island, NY, USA) containing $200 \mathrm{U} / \mathrm{mL}$ penicillin-streptomycin (Hyclone, Logan, UT, USA). After all visible connective tissue was removed, the isolated muscle tissue was finely minced and then digested in serum free Dulbecco's modified eagle medium/F12 (DMEM/F1, Hyclone) with $0.2 \%$ type-II collagenase (Invitrogen, Carlsbad, CA, USA) in a shaking water bath with $40 \mathrm{rpm}$ speeds for $2 \mathrm{~h}$ at $37^{\circ} \mathrm{C}$. The digest sample was filtered aseptically through 70 and $200 \mu \mathrm{m}$ steel mesh filters to isolate digested cells. Then the filtered cells were rinsed three times with serum free DMEM/F12 medium and then centrifuged twice at $1500 \times \mathrm{g}$ for $10 \mathrm{~min}$. Subsequently, cells were seeded at a density $6 \times 10^{5}$ per $60 \mathrm{~mm}$ culture dish in DMEM/F12 medium with $10 \%$ fetal bovine (FBS, Gibco) and penicillin-streptomycin and cultured at $37^{\circ} \mathrm{C}$ with $5 \% \mathrm{CO}_{2}$. After $1 \mathrm{~h}$, cells were washed with DMEM/F12 medium to wipe off un-adhered cells. After reaching $80 \%$ confluence, cells were digested with $0.05 \%$ trypsin and plated at a density of $5 \times 10^{4}$ cells $/ \mathrm{cm}$ in a 6-well plate for further research.

To induce differentiation, the above cells were cultured in induction medium containing DMEM/F12, 10\% FBS, $100 \mathrm{U} / \mathrm{mL}$ penicillin-streptomycin, $1 \mathrm{nM}$ dexamethasone (DEX, Sigma, St. Louis, MO, USA), $5 \mathrm{ng} / \mathrm{mL}$ insulin (Sigma), and $0.5 \mathrm{mM}$ 3-isobutyl 1-methylxanthine (IBMX, Sigma). After two days, the medium was changed with DMEM/F12 containing $10 \% \mathrm{FBS}, 100 \mathrm{U} / \mathrm{mL}$ penicillin-streptomycin, and $5 \mathrm{ng} / \mathrm{mL}$ insulin. The medium was changed every two days until day 8 .

\subsection{Cells Transfection}

To evaluate the effect of miR-125a-5p on the proliferation and differentiation of porcine intramuscular preadipocytes, cells were transfected respectively with synthetic miR-125a-5p mimics (sequence: 5'-UCCCUGAGACCCUUUAACCUGU-3'), inhibitors (sequence: 5'- ACAGGUUAAAGGGUCUCAGGGA-3'), or negative control (NC, sequence: 5'-UUUGUACUACACAAAAGUACUG-3' 5 $^{\prime}$-CAGUACUUUUGUGUAGUACAAA-3') (all purchased from RIBOBIO, Guangzhou, China). For proliferation, when the density of cells in 96-well plates reached 40\%, miR-125a-5p mimics ( $50 \mathrm{nM})$, inhibitors $(100 \mathrm{nM})$, or NC ( $50 \mathrm{nM})$ were transfected into cells using Lipofectamine2000 (Invitrogen) and Opti-MEM (Gibco) culture medium, according to the manufacturer's instructions. For differentiation, when the density of cells in 12-well plates reached $80 \%$, using the same way, miR-125a-5p mimics, inhibitors, or NC were transfected into cells according to the manufacturer's instructions, and the culture medium was changed to differentiation medium after $24 \mathrm{~h}$ of transfection.

\subsection{Proliferation Analysis by CCK8 and EdU Assays}

Porcine intramuscular preadipocytes seeded in 96-well plates in the DMEM/F12 medium supplement with $10 \%$ FBS were transfected with miR-125a-5p mimics, inhibitors, and NC. 
Cell proliferation was measured at $0,12,24,48$, and $72 \mathrm{~h}$ of transfection by using the Cell Counting kit 8 (CCK-8, Beyotime, Shanghai, China) according to the manufacturer's protocol. For 5-ethynyl-2'-deoxyuridine analysis (EdU, RIBOBIO, Guangzhou, China), cells were treated with $10 \mu \mathrm{M}$ EdU for $24 \mathrm{~h}$ after transfection and incubated for a further $10 \mathrm{~h}$. EdU staining was performed according to the manufacturer's protocol. Images were captured using an Olympus IX53 microscope (Olympus, Tokyo, Japan).

\subsection{Oil Red O Staining and Triglyceride Analysis}

Cells treated for 8 days were washed three times with PBS and fixed in $4 \%$ paraformaldehyde solution and incubated with $0.5 \%$ Oil Red $\mathrm{O}$ for $1 \mathrm{~h}$. After cell samples were further washed three times with PBS, images were captured using an Olympus IX53 microscope (Olympus). To measure triglyceride (TG) contents, stained cells were eluted with isopropanol for $20 \mathrm{~min}$ and the optical density (OD) values were detected with a spectrophotometer at a wavelength of $510 \mathrm{~nm}$, as described by our previous report $[22,23]$.

\subsection{Immunocytochemical Analysis}

As described by Du et al. [22], cell samples were washed three times with PBS, fixed in $4 \%$ paraformaldehyde for $30 \mathrm{~min}$, and then permeabilized with $0.5 \%$ Triton X-100 prior to blocking in 2\% goat serum. Subsequently, the above cell samples were incubated with an anti-adiponectin antibody (Bioss, Beijing, China, catalog number: bs-0471R) at $4{ }^{\circ} \mathrm{C}$ for $24 \mathrm{~h}$. This was followed by fluorescent secondary antibodies at $37^{\circ} \mathrm{C}$ for $1 \mathrm{~h}$. Images were captured using an Olympus IX53 microscope (Olympus).

\subsection{RNA Extraction and $q R T-P C R$}

Total RNA from cells and muscle tissues were extracted using a TRIzol Reagent (Invitrogen) according to the manufacturer's instructions. The RNA quality and concentration were estimated using denatured gel electrophoresis and a spectrophotometer (Thermo, Waltham, MA, USA), respectively. Subsequently, cDNA was synthesized and quantitative real-time PCR (qRT-PCR) was performed by the SYBR Premix Ex Taq kit (TaKaRa; LianDa, China) on a CFX96 system (Bio-Rad, Hercules, CA, USA). Relative expression levels of mRNAs and microRNAs were calculated using the $2^{-\Delta \Delta C t}$ method [48]. The primer sequences used for qRT-PCR are listed in Supplementary Table S1. U6 and $\beta$-actin were used as endogenous control genes for miRNA and mRNA, respectively.

\subsection{Mitochondrial Content}

The relative number of mitochondria was determined by measuring the ratio of mtDNA:nDNA as previously described [49]. The relative levels of mtDNA and nDNA were quantified using primers specific for mitochondrial cytochrome coxidase subunit 1 (Cox1) and the nuclear gene glucagon (GCG).

\subsection{Luciferase Reporter Assay}

The wild-type $3^{\prime}$ UTR of KLF13 and ELOVL6 (WT-KLF13 and WT-ELOVL6), Mutant-type 3'UTR of KLF13, and ELOVL6 (Mut-KLF13 and Mut-ELOVL6) were cloned into psiCHECKTM-2 vector at the $3^{\prime}$-end of the Renilla gene, which was made by a manufacturer (TsingKe Biotech, Chengdu, China). For luciferase reporter analysis, wild-type $3^{\prime}$ UTR or mutant-type $3^{\prime} U T R$ were co-transfected respectively with miR-125a-5p mimics, inhibitors, or NC into porcine intramuscular preadipocytess using Lipofectamine3000 (Invitrogen). Cells were harvested at $48 \mathrm{~h}$ post-transfection and luciferase activities were measured with the Dual-Glo Luciferase Assay System (Promega, Madison, WI, USA) following the manufacturer's instructions. 


\subsection{Measurement of IMF and Fatty Acid Composition}

The above muscle tissues stored at $-20{ }^{\circ} \mathrm{C}$ were studied in triplicate (each sample of $3 \mathrm{~g}$ ) and then IMF measurement was performed and determined as the percentage of fat extracted $3 \mathrm{~g}$ of fresh tissues using the Soxhlet petroleum-ether extraction method, as described by a previous study [50]. Furthermore, fatty acids were analyzed according to previously published protocols [51]. After porcine intramuscular preadipocytess were induced, differentiated, and transfected with miR-125a-5 mimics or NC for 8 days, $50 \mathrm{mg}$ cells samples were collected to determine fatty acids composition, which was completed by the Beijing Municipal Science and Technology limited company.

\subsection{Statistical Analysis}

All data are expressed as means $\pm \mathrm{SE}$, and statistical analyses were performed using SPSS22.0 software (SPSS22.0, SPSS Science, Chicago, IL, USA). Differences between groups were analyzed by applying Student's two-tailed $t$-test for two parametric groups and one-way analysis for the three least parametric groups. A value of $p<0.05$ indicated a significant difference.

\section{Conclusions}

In the present study, we identified a novel epigenetic regulator of porcine intramuscular fat content and composition. The results showed that expression levels of miR-125a-5p in porcine muscle tissues were negatively associated with IMF content, overexpression, or inhibition of miR-125a-5p along with promoted or repressed proliferation of porcine intramuscular preadipocytes, respectively. By directly targeting KLF13, miR-125a-5p inhibited the differentiation of porcine intramuscular preadipocytes. Particularly, we found that miR-125a-5p affected fatty acid composition by negatively regulating ELOVL6, a regulator of fatty acid composition. The data indicated miR-125a-5p may play an import role in porcine intramuscular adipogenesis and fatty acid composition regulation in porcine IMF.

Supplementary Materials: Supplementary materials can be found at http:/ / www.mdpi.com/1422-0067/19/2/ $501 /$ s1.

Acknowledgments: The study was supported by the Sichuan Sci \& Tech Support Program (Pig Genetic Resources Exploitation and Utilization for the 13th Five-Year-Project, No. 16ZC2838) the Chinese National Sci \& Tech Support Program (No. 2015BAD03B01), and the earmarked fund for China Agriculture Research System (No. CARS-36-05B).

Author Contributions: Jingjing Du, Shunhua Zhang, and Li Zhu conceived and designed the experiments; Jingjing Du, Yan Xu, Peiwen Zhang, Xue Zhao, Mailin Gan, Qiang Li, Jideng Ma, and Guoqing Tang performed the experiments; Yanzhi Jiang, Jinyong Wang, and Xuewei Li analyzed the data; Jingjing Du wrote the paper.

Conflicts of Interest: The authors declare no conflict of interest.

$\begin{array}{ll}\text { Abbreviations } \\ \text { CDK2 } & \text { Cyclin-dependent kinase 2 } \\ \text { Cyclin B } & \text { Cell cycle protein B } \\ \text { CDK3 } & \text { Cyclin-dependent kinases } 3 \\ \text { CDK4 } & \text { Cyclin-dependent kinases } 4 \\ \text { p21 } & \text { Cyclin-dependent kinase inhibitor } 1 \\ \text { FABP4 } & \text { Adipocyte fatty acid-binding protein } 4 \\ \text { FASN } & \text { Fatty acid synthase } \\ \text { PPAR } \gamma & \text { Peroxisome proliferator activated receptor } \gamma \\ \text { FATP4 } & \text { Fatty acid transporter member } 4 \\ \text { C/EBP } \alpha & \text { CCAAT/enhancer binding protein } \alpha \\ \text { Tfam } & \text { Transcription factor A } \\ \text { Mterf1 } & \text { Mitochondrial transcription termination factor } 1 \\ \text { COX5b } & \text { Cytochrome c oxidase subunit Vb } \\ \text { TMEM70 } & \text { Transmembrane protein } 70 \\ \text { COX8b } & \text { Cytochrome c oxidase subunit VIIIb }\end{array}$




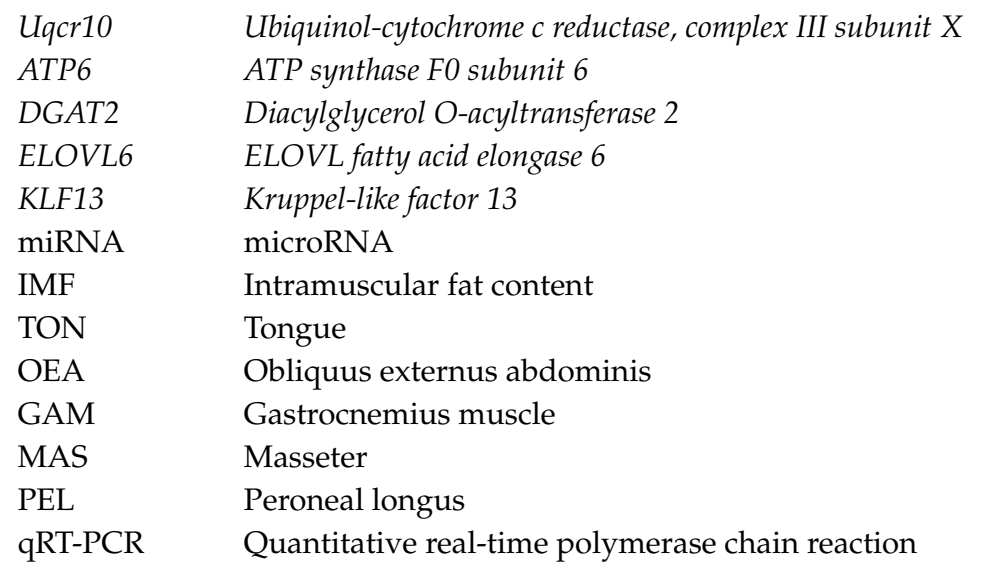

\section{References}

1. Xiong, Z.; Sun, D.W.; Pu, H.; Gao, W.; Dai, Q. Applications of emerging imaging techniques for meat quality and safety detection and evaluation: A review. Crit. Rev. Food Sci. Nutr. 2017, 57, 755-768. [CrossRef] [PubMed]

2. Lunney, J.K.; Eguchiogawa, T.; Uenishi, H.; Wertz, N.; Butler, J.E.; Rothschild, M.F.; Ruvinsky, A. The Genetics of the Pig; CABI: Wallingford, UK, 2011.

3. Kim, J.M.; Lim, K.S.; Ko, K.B.; Ryu, Y.C. Estimation of pork quality in live pigs using biopsied muscle fibre number composition. Meat Sci. 2017, 137, 130-133. [CrossRef] [PubMed]

4. Hocquette, J.F.; Gondret, F.; Baéza, E.; Médale, F.; Jurie, C.; Pethick, D.W. Intramuscular fat content in meat-producing animals: Development, genetic and nutritional control, and identification of putative markers. Animal 2010, 4, 303-319. [CrossRef] [PubMed]

5. Fernandez, X.; Monin, G.; Talmant, A.; Mourot, J.; Lebret, B. Influence of intramuscular fat content on the quality of pig meat. I. Composition of the lipid fraction and sensory characteristics of. Meat Sci. 1999, 53, 59-65. [CrossRef]

6. Gerbens, F.; Verburg, F.J.; Van Moerkerk, H.T.; Engel, B.; Buist, W.; Veerkamp, J.H.; te Pas, M.F. Associations of heart and adipocyte fatty acid-binding protein gene expression with intramuscular fat content in pigs. J. Anim. Sci. 2001, 79, 347-354. [CrossRef] [PubMed]

7. Shahidi, F. Lipid-Derived Flavors in Meat Products. In Meat Processing: Improving Quality; Kerry, J.P., Kerry, J.F., et al., Eds.; Woodhead Publishing: Cambridge, UK, 2002; pp. 105-121.

8. Williams, C.M. Dietary fatty acids and human health. Ann. Zootech. 2000, 49, 165-180. [CrossRef]

9. Ravnskov, $\mathrm{U}$. The questionable role of saturated and polyunsaturated fatty acids in cardiovascular disease. J. Clin. Epidemiol. 1998, 51, 443-460. [CrossRef]

10. Kris-Etherton, P.M.; Guixiang Zhao, M.S.; Pelkman, C.L.; Ms, V.K.F.; Coval, S.M. Beneficial effects of a diet high in monounsaturated fatty acids on risk factors for cardiovascular disease. Nutr. Clin. 2000, 3, 153-162. [CrossRef]

11. Bartel, D.P. MicroRNAs: Genomics, biogenesis, mechanism, and function. Cell 2004, 116, 281-297. [CrossRef]

12. Bushati, N.; Cohen, S.M. MicroRNA functions. Annu. Rev. Cell. Dev. Biol. 2007, 23, 175-205. [CrossRef] [PubMed]

13. Krol, J.; Loedige, I.; Filipowicz, W. The widespread regulation of microRNA biogenesis, function and decay. Nat. Rev. Genet. 2010, 11, 597-610. [CrossRef] [PubMed]

14. Li, H.; Zhang, Z.; Zhou, X.; Wang, Z.; Wang, G.; Han, Z. Effects of microRNA-143 in the differentiation and proliferation of bovine intramuscular preadipocytes. Mol. Biol. Rep. 2011, 38, 4273-4280. [CrossRef] [PubMed]

15. Guo, Y.; Chen, Y.; Yun, Z.; Yue, Z.; Chen, L.; Mo, D. Up-regulated miR-145 expression inhibits porcine preadipocytes differentiation by targeting irs1. Int. J. Biol. Sci. 2012, 8, 1408-1417. [CrossRef] [PubMed]

16. Sun, Y.M.; Qin, J.; Liu, S.G.; Cai, R.; Chen, X.C.; Wang, X.M.; Pang, W.J. PDGFR $\alpha$ regulated by miR-34a and FoxO1 promotes adipogenesis in porcine intramuscular preadipocytes through Erk signaling pathway. Int. J. Mol. Sci. 2017, 18. [CrossRef] [PubMed] 
17. Diawara, M.R.; Hue, C.; Wilder, S.P.; Venteclef, N.; Aronwisnewsky, J.; Scott, J.; Clement, K.; Gauguier, D.; Calderari, S. Adaptive expression of microRNA-125a in adipose tissue in response to obesity in mice and men. PLoS ONE 2014, 9, e91375. [CrossRef] [PubMed]

18. Ji, H.L.; Song, C.C.; Li, Y.F.; He, J.J.; Li, Y.L.; Zheng, X.L.; Yang, G.S. miR-125a inhibits porcine preadipocytes differentiation by targeting err $\alpha$. Mol. Cell. Biochem. 2014, 395, 155-165. [CrossRef] [PubMed]

19. Hong-Lei, J.I.; Song, C.C.; You-Lei, L.I.; Yue-Feng, L.I.; Zheng, X.L.; Yang, G.S. miR-125a-5p is implicated in 3t3-11 preadipocytes differentiation. Chin. J. Biochem. Mol. Biol. 2014, 30, 1147-1154.

20. Gareri, C.; Iaconetti, C.; Sorrentino, S.; Covello, C.; De, R.S.; Indolfi, C. miR-125a-5p modulates phenotypic switch of vascular smooth muscle cells by targeting ets-1. J. Mol. Biol. 2017, 429, 1817-1828. [CrossRef] [PubMed]

21. Liu, C.; Shen, L.; Du, J.; Wu, X.; Luo, J.; Pu, Q.; Tan, Z.; Cheng, X.; Du, J.; Yang, Q. The effect of lipid metabolism-related genes on intramuscular fat content and fatty acid composition in multiple muscles. Anim. Prod. Sci. 2017. [CrossRef]

22. Li, M.; Wu, H.; Luo, Z.; Xia, Y.; Guan, J.; Wang, T.; Gu, Y.; Chen, L.; Zhang, K.; Ma, J. An atlas of DNA methylomes in porcine adipose and muscle tissues. Nat. Commun. 2012, 3, 850. [CrossRef] [PubMed]

23. Cui, J.X.; Zeng, Y.Q.; Wang, H.; Chen, W.; Du, J.F.; Chen, Q.M.; Hu, Y.X.; Yang, L. The effects of dgat1 and dgat2 mrna expression on fat deposition in fatty and lean breeds of pig. Livest. Sci. 2011, 140, $292-296$. [CrossRef]

24. Revilla, M.; Ramayocaldas, Y.; Castelló, A.; Corominas, J.; Puigoliveras, A.; Ibáñezescriche, N.; Muñoz, M.; Ballester, M.; Folch, J.M. New insight into the ssc8 genetic determination of fatty acid composition in pigs. Genet. Sel. Evol. 2014, 46, 28. [CrossRef] [PubMed]

25. Chen, F.F.; Xiong, Y.; Peng, Y.; Gao, Y.; Qin, J.; Chu, G.Y.; Pang, W.J.; Yang, G.S. miR-425-5p inhibits differentiation and proliferation in porcine intramuscular preadipocytes. Int. J. Mol. Sci. 2017, 18, 2101. [CrossRef] [PubMed]

26. Tsai, L.H.; Lees, E.; Faha, B.; Harlow, E.; Riabowol, K. The cdk2 kinase is required for the g1-to-s transition in mammalian cells. Oncogene 1993, 8, 1593-1602. [PubMed]

27. Sherr, C.J.; Roberts, J.M. Inhibitors of mammalian g1 cyclin-dependent kinases. Genes Dev. 1995, 9, 1149-1163. [CrossRef] [PubMed]

28. Ito, M. Factors controlling cyclin b expression. Plant Mol. Biol. 2000, 43, 677-690. [CrossRef] [PubMed]

29. Harper, J.W.; Adami, G.R.; Wei, N.; Keyomarsi, K.; Elledge, S.J. The p21 cdk-interacting protein cip1 is a potent inhibitor of g1 cyclin-dependent kinases. Cell 1993, 75, 805-816. [CrossRef]

30. Alaynick, W.A. Nuclear receptors, mitochondria and lipid metabolism. Mitochondrion 2008, 8, 329-337. [CrossRef] [PubMed]

31. Civitarese, A.E.; Smith, S.R.; Ravussin, E. Diet, energy metabolism and mitochondrial biogenesis. Curr. Opin. Clin. Nutr. 2007, 10, 679-687. [CrossRef] [PubMed]

32. Medina-Gómez, G. Mitochondria and endocrine function of adipose tissue. Best Pract. Res. Clin. Endocrinol. Metab. 2012, 26, 791-804. [CrossRef] [PubMed]

33. Xu, D.; Gao, Y.; Hu, N.; Wu, L.; Chen, Q. miR-365 ameliorates dexamethasone-induced suppression of osteogenesis in MC3T3-E1 cells by targeting HDAC4. Int. J. Mol. Sci. 2017, 18, 977. [CrossRef] [PubMed]

34. Wang, Y.; Yang, T.; Liu, Y.; Zhao, W.; Zhang, Z.; Lu, M.; Zhang, W. Decrease of miR-195 promotes chondrocytes proliferation and maintenance of chondrogenic phenotype via targeting fgf-18 pathway. Int. J. Mol. Sci. 2017, 18, 975. [CrossRef] [PubMed]

35. Zhang, B.W.; Cai, H.F.; Wei, X.F.; Sun, J.J.; Lan, X.Y.; Lei, C.Z.; Lin, F.P.; Qi, X.L.; Martin, P.; Hong, C. miR-30-5p regulates muscle differentiation and alternative splicing of muscle-related genes by targeting mbnl. Int. J. Mol. Sci. 2016, 17, 182. [CrossRef] [PubMed]

36. Jiang, S.; Wei, H.; Song, T.; Yang, Y.; Zhang, F.; Zhou, Y.; Peng, J.; Jiang, S. Klf13 promotes porcine adipocyte differentiation through ppary activation. Cell Biosci. 2015, 5, 1-14. [CrossRef] [PubMed]

37. Alonso, V.; Campo, M.M.; Español, S.; Roncalés, P.; Beltrán, J.A. Effect of crossbreeding and gender on meat quality and fatty acid composition in pork. Meat Sci. 2009, 81, 209-217. [CrossRef] [PubMed]

38. Aïtkaddour, A.; Thomas, A.; Mardon, J.; Jacquot, S.; Ferlay, A.; Gruffat, D. Potential of fluorescence spectroscopy to predict fatty acid composition of beef. Meat Sci. 2016, 113, 124-131. [CrossRef] [PubMed] 
39. Green, C.D.; Ozgudenakkoc, C.G.; Wang, Y.; Jump, D.B.; Olson, L.K. Role of fatty acid elongases in determination of de novo synthesized monounsaturated fatty acid species. J. Lipid Res. 2010, 51, 1871-1877. [CrossRef] [PubMed]

40. Moon, Y.A.; Shah, N.A.; Mohapatra, S.; Warrington, J.A.; Horton, J.D. Identification of a mammalian long chain fatty acyl elongase regulated by sterol regulatory element-binding proteins. J. Biol. Chem. 2001, 276, 45358-45366. [CrossRef] [PubMed]

41. Pascual, J.V.; Rafecas, M.; Canela, M.A.; Boatella, J.; Bou, R.; Barroeta, A.C.; Codony, R. Effect of increasing amounts of a linoleic-rich dietary fat on the fat composition of four pig breeds. Food Chem. 2007, 100, 1639-1648. [CrossRef]

42. Lorgeril, M.D.; Salen, P. New insights into the health effects of dietary saturated and omega- 6 and omega-3 polyunsaturated fatty acids. BMC Med. 2012, 10, 2-5. [CrossRef] [PubMed]

43. Ulbricht, T.L.V.; Southgate, D.A.T. Coronary heart disease: Seven dietary factors. Lancet 1991, 338, $985-992$. [CrossRef]

44. Jiménezcolmenero, F.; Ventanas, J.; Toldrá, F. Nutritional composition of dry-cured ham and its role in a healthy diet. Meat Sci. 2013, 84, 585-593. [CrossRef] [PubMed]

45. Kallas, Z.; Realini, C.E.; Gil, J.M. Health information impact on the relative importance of beef attributes including its enrichment with polyunsaturated fatty acids (omega-3 and conjugated linoleic acid). Meat Sci. 2014, 97, 497-503. [CrossRef] [PubMed]

46. Zhao, C.; Chen, X.; Wu, W.; Wang, W.; Pang, W.; Yang, G. Mat2b promotes adipogenesis by modulating same levels and activating akt/erk pathway during porcine intramuscular preadipocyte differentiation. Exp. Cell Res. 2016, 344, 11-21. [CrossRef] [PubMed]

47. Peng, Y.; Chen, F.F.; Ge, J.; Zhu, J.Y.; Shi, X.; Li, X.; Yu, T.Y.; Chu, G.Y.; Yang, G.S. miR-429 inhibits differentiation and promotes proliferation in porcine preadipocytes. Int. J. Mol. Sci. 2016, 17, 2047. [CrossRef] [PubMed]

48. Livak, K.J.; Schmittgen, T.D. Analysis of relative gene expression data using real-time quantitative pcr and the $2^{-\Delta \Delta C t}$ method. Methods 2001, 25, 402-408. [CrossRef] [PubMed]

49. Yan, M.; Audet-Walsh, Ã.; Manteghi, S.; Dufour, C.R.; Walker, B.; Baba, M.; St-Pierre, J.; Giguã Re, V.; Pause, A. Chronic ampk activation via loss of flcn induces functional beige adipose tissue through pgc- $1 \alpha / E R R \alpha$. Genes Dev. 2016, 30, 1034-1046. [CrossRef] [PubMed]

50. Gandolfi, G.; Mazzoni, M.; Zambonelli, P.; Lalatta-Costerbosa, G.; Tronca, A.; Russo, V.; Davoli, R. Perilipin 1 and perilipin 2 protein localization and gene expression study in skeletal muscles of european cross-breed pigs with different intramuscular fat contents. Meat Sci. 2011, 88, 631-637. [CrossRef] [PubMed]

51. Tan, Z.; Du, J.; Shen, L.; Liu, C.; Ma, J.; Bai, L.; Jiang, Y.; Tang, G.; Li, M.; Li, X. miR-199a-3p affects adipocytes differentiation and fatty acid composition through targeting scd. Biochem. Biophys. Res. Commun. 2017, 492, 82-88. [CrossRef] [PubMed]

(C) 2018 by the authors. Licensee MDPI, Basel, Switzerland. This article is an open access article distributed under the terms and conditions of the Creative Commons Attribution (CC BY) license (http://creativecommons.org/licenses/by/4.0/). 